\title{
Satellite potentials of motor unit action potentials in normal muscles: a new hypothesis for their origin
}

\author{
Zoia C. Lateva*, Kevin C. McGill \\ Rehabilitation Research and Development Center, VA Palo Alto Health Care System, 3801 Miranda Avenue/153, Palo Alto, CA 94304-1200, USA
}

Accepted 12 April 1999

\begin{abstract}
Objective: A satellite potential is a late component of the motor unit action potential (MUAP) that occurs both in pathologic and normal muscle. We investigated the physiological mechanisms responsible for satellite potentials in normal muscle by relating the latencies of MUAP features to the timing of the underlying electrical events.

Methods: We analyzed 21 MUAPs with satellite potentials that had been recorded using a monopolar needle electrode from brachial biceps and tibialis anterior muscles in 10 normal subjects. We estimated the endplate-to-electrode, endplate-to-tendon, and satellite propagation times from the latencies, with respect to the MUAP onset, of the MUAP spike, terminal wave, and satellite.

Results: Satellite latencies ranged from 8.8 to $32 \mathrm{~ms}$, too long to be explained by mechanisms involving regenerating axons or atrophic muscle fibers. The spike-to-satellite time intervals approximated either twice the spike-to-terminal-wave interval (17 MUAPs) or twice the terminal-wave latency (4 MUAPs).

Conclusions: These results are consistent with the hypothesis that satellite potentials are due to retrograde propagation in a non-innervated muscle fiber that is connected with an innervated muscle fiber at one of the muscle/tendon junctions. Such a configuration could arise as a result of longitudinal muscle-fiber splitting. (C) 1999 Elsevier Science Ireland Ltd. All rights reserved.
\end{abstract}

Keywords: Needle electromyography; Motor unit action potential; Satellite potential; Muscle fiber longitudinal splitting; Velocity recovery function

\section{Introduction}

Satellite potentials (or late-latency components) are a recognized phenomenon in motor unit action potential (MUAP) analysis (Stålberg et al., 1986, 1996; Daube, 1991). The most commonly accepted definition for the satellite potential is that it is a late spike, usually an action potential (AP) from a single muscle fiber, distinct from the main spike but time-locked to it (Stålberg et al., 1986). In some rare instances, time-locked potentials have been reported to appear before the main spike (Lang and Partanen, 1976; Stålberg et al., 1986; Finsterer and Mamoli, 1997). Satellite potentials are most commonly seen in some neuropathies and myopathies (Borenstein and Desmedt, 1975, 1980; Lang and Partanen, 1976; Uncini et al., 1990; Cruz-Martinez and Lopez-Terradas, 1992; Finsterer and Mamoli, 1997; Rowinska-Marcinska et al., 1997). Satellite potentials are also occasionally seen in healthy muscles (Buchthal and Rosenfalck, 1973; Borenstein and Desmedt,

\footnotetext{
* Corresponding author. Tel.: + 1-650-493-5000, ext.: 63048; fax: + 1650-493-4919.

E-mail address: lateva@roses.stanford.edu (Z.C. Lateva)
}

1975; Lang and Partanen, 1976; Finsterer and Mamoli, 1997).

All of the previously proposed explanations for the occurrence of satellite potentials involve pathological mechanisms leading to an increased AP propagation time along regenerating axons and their branches and/or along atrophic muscle fibers. This is based on the existence in pathological muscle of extremely long and/or slender (possibly unmyelinated) collateral axon sprouts; aberrant endplate positions because of collateral reinnervation; immature endplates with slow neuromuscular transmission; and small diameter muscle fibers resulting from atrophy, fragmentation after focal necrosis, or regeneration. However, while satellite potentials may be caused by one or more of these mechanisms in cases of neuromuscular disorders, it is not clear that such mechanisms are necessarily responsible for the satellite potentials in normal muscles.

This paper presents a detailed analysis of the waveforms of several MUAPs with satellite potentials from normal muscles of healthy subjects. The analysis is based on the results from previous simulation and experimental studies (Dimitrova, 1974; Stålberg et al., 1986, 1996; Dimitrova et al., 1991; Gootzen et al., 1991; Lateva et al., 1993, 1996; 


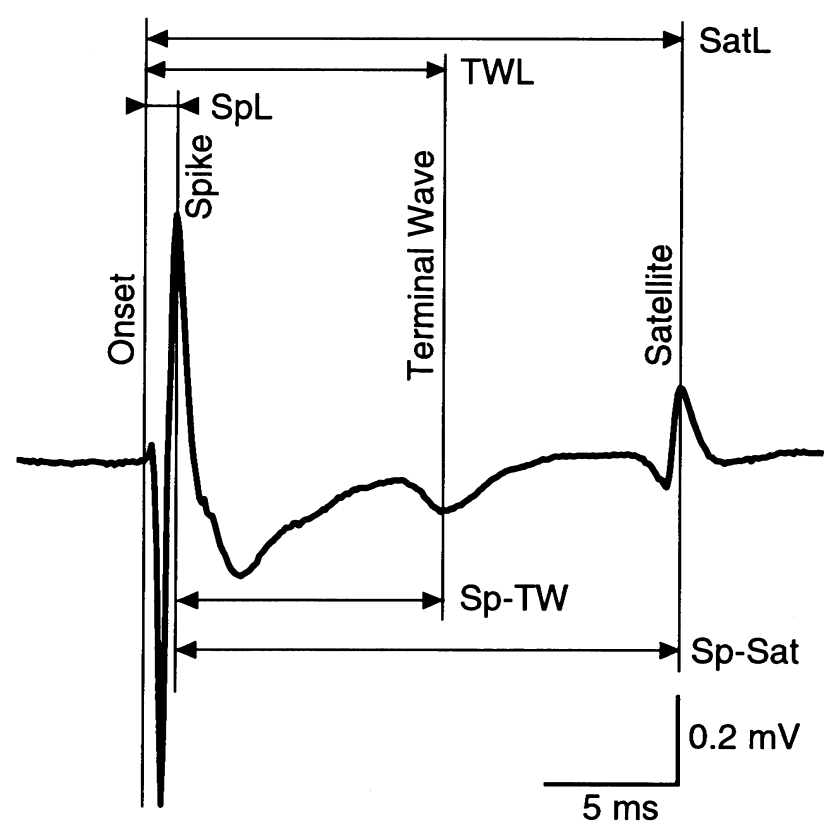

Fig. 1. Analysis of the MUAP waveform. First, 4 MUAP time markers were identified: onset, spike, terminal wave and satellite, which correspond, respectively to the following electrical events: initiation of the MUAP at the endplate, arrival of the MUAP at the recording electrode, arrival of the MUAP at the muscle/tendon junction and arrival of the satellite potential at the recording electrode. Second, the following latencies and intervals were measured: spike latency ( $\mathrm{SpL})$, terminal-wave latency (TWL), satellite latency (SatL), spike-terminal-wave interval (Sp-TW) and spike-satellite interval (Sp-Sat).

Lateva and McGill, 1998a,b) that relate specific morphological features of the MUAP to certain electrical events in the muscle. In particular, in MUAPs recorded by monopolar needle electrodes, the 4 main stages of electrical activity the initiation, propagation, termination, and slow repolarization - are each marked by a specific morphological feature. The initiation of electrical activity is marked by the distinct onset of the MUAP (Stålberg et al., 1996; Lateva and McGill, 1998a,b). Since the single-fiber action potentials (SFAPs) are initiated at the endplates of the individual muscle fibers of the MU almost simultaneously (Buchtal, et al., 1955) and since the resulting electrical field spreads through the volume conductor instantaneously, the MUAP onset is recorded at the same time at all recording sites. The propagation of electrical activity past the recording electrode is marked by the spike of the MUAP. The latency of the spike thus depends on the location of the recording electrode with respect to the endplate and the muscle/tendon junction. The termination of electrical activity at the muscle/tendon junction is marked by a distinct MUAP deflection known as the terminal wave (Gydikov and Kosarov, 1972; Kosarov and Gydikov, 1975; Gootzen et al., 1991; Lateva et al., 1996; Roeleveld et al., 1997). Like the onset, the terminal wave occurs at the same time at all recording sites. The terminal wave has a finite duration owing to the duration of the intracellular action potential and the temporal dispersion of SFAP arrival times at the muscle/tendon junction. Finally, the slow repolarization of the muscle fibers has recently been shown to be responsible for the slow afterwave of the MUAP (Lateva and McGill, 1998a).

The MUAP waveform thus presents a temporal record of the sequence of electrical events within the MU. In particular, the onset-to-spike and onset-to-terminal-wave latencies are, respectively, direct measures of the endplate-to-electrode and endplate-to-tendon propagation times. This makes it possible to compare the propagation times of the satellite potential and the main MUAP component.

The results of the analysis lead us to propose a new hypothesis for the origin of satellite potentials in normal human muscles. The hypothesis states that satellite potentials are produced by pairs of muscle fibers that are connected at one of the muscle/tendon junctions. One of the fibers receives neural innervation while the other does not. This configuration might arise because of longitudinal muscle-fiber splitting (Bell and Conen, 1968; Swash and Schwartz, 1977; Editorial, 1978; Swash and Schwartz, 1984; Loughlin, 1993).

\section{Materials and methods}

We searched for MUAPs with satellite potentials in 516 EMG signals that were recorded as a part of a previous study (Howard et al., 1988). The EMG signals were recorded from brachial biceps (BB) and tibialis anterior (TA) muscles of 10 normal volunteers (6 males and 4 females) aged 20-37 years (mean 27) with no history of neuromuscular disease and with normal EMG exams. All subjects gave informed consent to the experimental procedure which was approved by the Stanford University Committee on the Use of Human Subjects in Research.

The signals were recorded intramuscularly by a monopolar needle electrode (TECA MF25) during voluntary isometric contractions at less than $30 \%$ MVC. The surface reference electrode was put close to the insertion site. Signals from monopolar needle electrodes were investigated because they yield MUAPs in which the onset and terminal wave can be clearly seen. In signals recorded by concentric needle electrodes, on the other hand, these components are often canceled by the contribution from the cannula (Stålberg et al., 1986, 1996). Ten needle insertions in different parts of each muscle were performed and 3 10-s-long EMG signals were collected at each insertion site at different force levels. The signals were filtered at $8 \mathrm{~Hz}$ and $8 \mathrm{kHz}$, sampled at $10 \mathrm{kHz}$, stored on tape, and then transferred to a Macintosh computer for further analysis.

The MUAP trains in each EMG signal were identified offline using a computerized decomposition program that we developed. The program was able to identify many of the MUAPs and MUAP discharges automatically using the ADEMG algorithm (McGill et al., 1985; McGill and Dorfman, 1989). Then one of the investigators checked the entire 
Table 1

Profile of subjects

\begin{tabular}{|c|c|c|c|c|c|c|}
\hline \multirow[t]{2}{*}{ Subject } & \multirow[t]{2}{*}{ Age (years) } & \multirow[t]{2}{*}{ Sex } & \multicolumn{2}{|c|}{ MUAPs in BB } & \multicolumn{2}{|c|}{ MUAPs in TA } \\
\hline & & & Total & With satellites & Total & With satellites \\
\hline 1 & 25 & $\mathrm{~F}$ & 256 & 0 & 198 & 0 \\
\hline 2 & 24 & $\mathrm{~F}$ & 246 & 1 & 273 & 2 \\
\hline 3 & 28 & M & 231 & 0 & 232 & 2 \\
\hline 4 & 37 & $\mathrm{~F}$ & 192 & 9 & 177 & 0 \\
\hline 5 & 20 & M & 190 & 0 & 186 & 0 \\
\hline 6 & 26 & M & 161 & 0 & 138 & 0 \\
\hline 7 & 30 & M & 243 & 0 & 251 & 3 \\
\hline 8 & 27 & $\mathrm{~F}$ & 236 & 0 & 213 & 0 \\
\hline 9 & 28 & M & 214 & 0 & 196 & 1 \\
\hline 10 & 32 & M & 218 & 2 & 201 & 1 \\
\hline Total & & & 2187 & 12 & 2065 & 9 \\
\hline
\end{tabular}

EMG signal using the program interactively to verify the results and identify any discharges that remained. Finally, the MUAP waveforms were averaged from the signal using an interference-cancellation algorithm (McGill et al., 1985). In this way it was possible to obtain MUAP waveforms with flat baselines and very low noise levels, in which the onsets and terminal waves could usually be clearly recognized.

The MUAP waveforms were inspected for satellite potentials. Signal components were regarded as satellites if they were time-locked to the main MUAP spike and occurred before the MUAP onset or after the MUAP terminal wave. The MUAP averaging window extended from $40 \mathrm{~ms}$ before, to $40 \mathrm{~ms}$ after the main spike so that any time-locked activity within these limits could be detected. These limits were larger than the maximum spike-satellite interval (26 ms) reported in normal muscle by Lang and Partanen (1976).

For each MUAP found to have a satellite potential, a detailed analysis of the averaged MUAP waveform was performed. First, the important time markers were identified: the onset, the peak of the main spike, the peak of the terminal wave and the peak of the satellite spike (Fig. 1). Then the following latencies were measured: (1) the spike latency; (2) the terminal wave latency; and (3) the satellite latency. From these measures, two more time intervals were derived: the spike-terminal-wave interval and the spikesatellite interval. The spike and terminal-wave latencies correspond to the propagation times of the main MUAP component from the endplate to the recording electrode and to the tendon, respectively. The spike-satellite interval equals the difference between the propagation times of the main MUAP component and the satellite potential along both the nerve twigs and muscle fibers from the point of axonal branching to the site of the recording electrode. The peak-to-peak (p-p) amplitudes of the main MUAP spike and the satellites were also measured. The durations of the terminal wave and the satellite spike were estimated.

Finally, the amount of blocking and the spike-satelliteinterval variability were determined. Each discharge of each relevant MUAP was reexamined to check for blocking of the satellite potential and to measure the spike-satellite interval. The measurements were made to sub-samplinginterval precision by high-pass filtering the signal (1.2 $\mathrm{kHz}$ ) and using a high-resolution alignment algorithm (McGill and Dorfman, 1984). The interdischarge intervals (IDIs) were also measured. The jitter of the spike-satellite interval was then computed as the mean consecutive difference between the intervals, using the correction method of Davis et al. (1988) to remove the IDI dependency attributable to the velocity recovery function.

\section{Results}

The EMG signals were moderate interference patterns with 3-16 MUAP trains (mean 8) and firing rates in the range of 5-18 Hz. Totals of 2187 and 2065 different MUAPs were obtained from the EMG signals recorded in $\mathrm{BB}$ and in TA, respectively. Among all the MUAPs there were only 21 with satellite potentials, of which 12 were in BB $(0.55 \%)$ and 9 in TA $(0.44 \%)$. The distribution of MUAPs with satellites between subjects is shown in Table 1 .

The p-p amplitude of the satellite potentials ranged from 50 to $581 \mu \mathrm{V}$ (mean $205 \mu \mathrm{V}$ ). The satellites were generally much smaller than the MUAP main spike. The ratio between the satellite p-p amplitude and the main-spike p-p amplitude was in the range of $0.05-0.7$ (mean 0.2). Measuring the exact duration of the satellite potentials was difficult because they were usually superimposed on the MUAP slow afterwave, but their estimated duration was not longer than that of a SFAP (fibrillation potential), i.e. about 2-3 ms.

No MUAPs had more than one satellite, and no satellites were observed before the MUAP onset. The satellite latencies ranged from 8.8 to $32.2 \mathrm{~ms}$ (mean $18.8 \mathrm{~ms}$ ). The spikesatellite intervals ranged from 6.3 to $21.9 \mathrm{~ms}$ (mean 15.5 $\mathrm{ms}$ ). There was no relationship between the spike latency and the spike-satellite interval (Fig. 2).

While analyzing the waveforms of the MUAPs with satellite potentials, we noticed that they could be divided into 


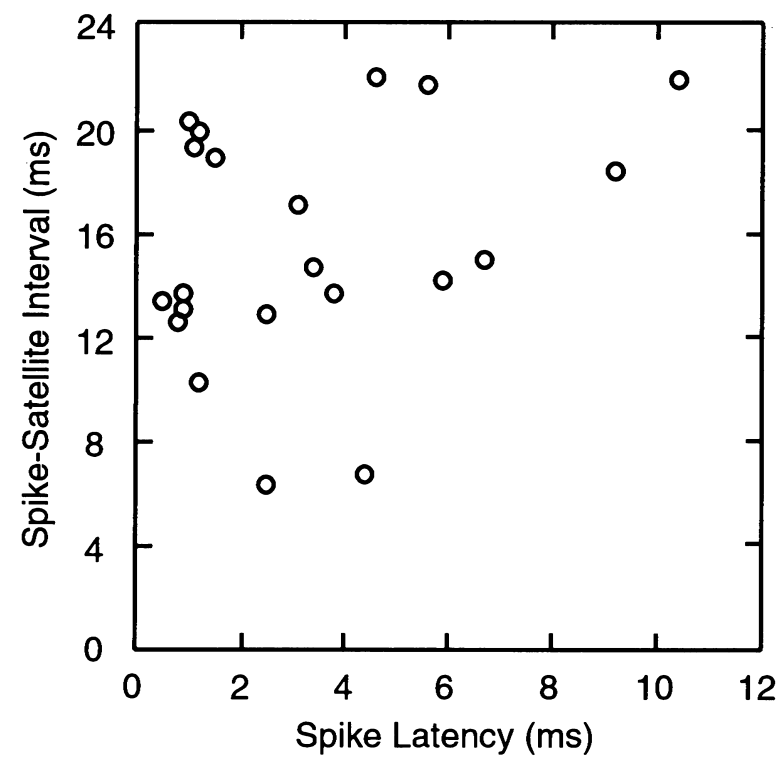

Fig. 2. No relationship existed between the spike-satellite interval and the spike latency for 21 MUAPs with satellite potentials.

two groups. The first group consisted of 17 MUAPs for which the spike-satellite interval was approximately twice as long as the spike-terminal-wave interval, so that the terminal wave occurred midway between the spike and the satellite (Fig. 3A). The second group included 4 MUAPs that had longer spike-satellite intervals, so that the terminal wave occurred closer to the spike than to the satellite (Fig. 4A). This result is presented in Fig. 5 for all 21 MUAPs. For the MUAPs in the first group, the midpoint between the spike and the satellite always fell within the extent of the terminal wave (which ranged from 2.5 to $4 \mathrm{~ms}$ ), and in fact always fell within $1 \mathrm{~ms}$ of the peak of the terminal wave (Fig. 5, open circles). For the MUAPs in the second group, the spike-satellite interval was always larger than twice the spike-terminal-wave interval (Fig. 5, filled circles) but it was approximately equal to twice the terminal-wave latency (Fig. 5, asterisks).

A schematic representation of the hypothesized physiological model that explains these results is given in Figs. 3B and $4 \mathrm{~B}$. The model shows a single fiber, representing the bulk of the MU, and a pair of fibers with an anatomical continuity at one muscle/tendon junction. During the MUAP, the rightward-going SFAP in the innervated fiber of the connected pair propagates to the point of continuity and then back along the non-innervated fiber, where, as it passes the electrode, it is recorded as the satellite potential. For the first group of MUAPs (Fig. 3), the electrode is located between the endplate and the point of continuity, so that the satellite latency equals the spike latency plus two times the spike-terminal-wave interval. Thus, in this case, the spike-satellite interval equals twice the spike-terminalwave interval. For the second group of MUAPs (Fig. 4), the electrode is located on the other side of the endplate, so that the satellite latency equals two times the terminal-wave latency (assuming that the left and right muscle-fiber semilengths are approximately equal) plus the spike latency. In this case, the spike-satellite interval equals twice the terminal-wave latency.

Blocking of the satellite potential was never observed in any of the 1660 recorded discharges of the 21 MUAPs. The spike-satellite intervals exhibited relatively little variability. The mean consecutive differences ranged from 18 to $200 \mu \mathrm{s}$, while the IDIs ranged from 53 to $518 \mathrm{~ms}$. A large part of the variability could be attributed to the velocity recovery function, as evidenced by the significant positive correlation

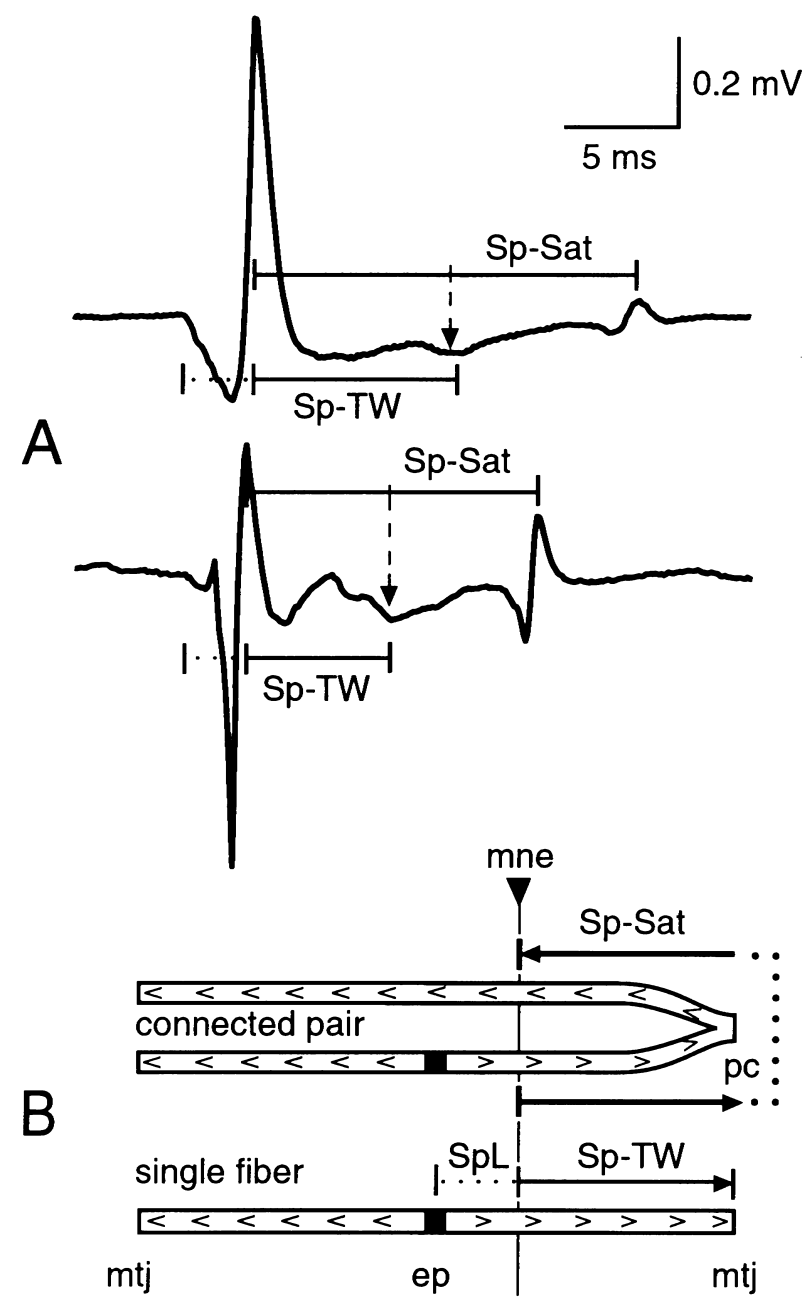

Fig. 3. In 17 of the MUAPs, the spike-satellite interval was approximately twice the spike-terminal-wave interval. (A) Example MUAPs from BB (top) and TA (bottom). Note that the midpoints of the spike-satellite intervals (arrows) occurred within the extent of the terminal waves. (B) Schematic representation of the proposed physiological model, showing a single fiber and a pair of fibers connected at the muscle/tendon junction (ep, endplate; mjt, muscle/tendon junction; mne, monopolar needle electrode; $\mathrm{pc}$, point of continuity). The direction of AP propagation along the fibers is shown by arrows. The satellite potential is hypothesized to be due to retrograde propagation in the non-innervated fiber of the connected pair. In this case, the electrode is located on the same side of the endplate as the point of continuity, so the satellite latency corresponds to the endplate-to-electrode propagation time $(\mathrm{SpL})$ plus two times the electrode-to-tendon propagation time (Sp-TW). Therefore, Sp-Sat $=2 *$ Sp-TW. 


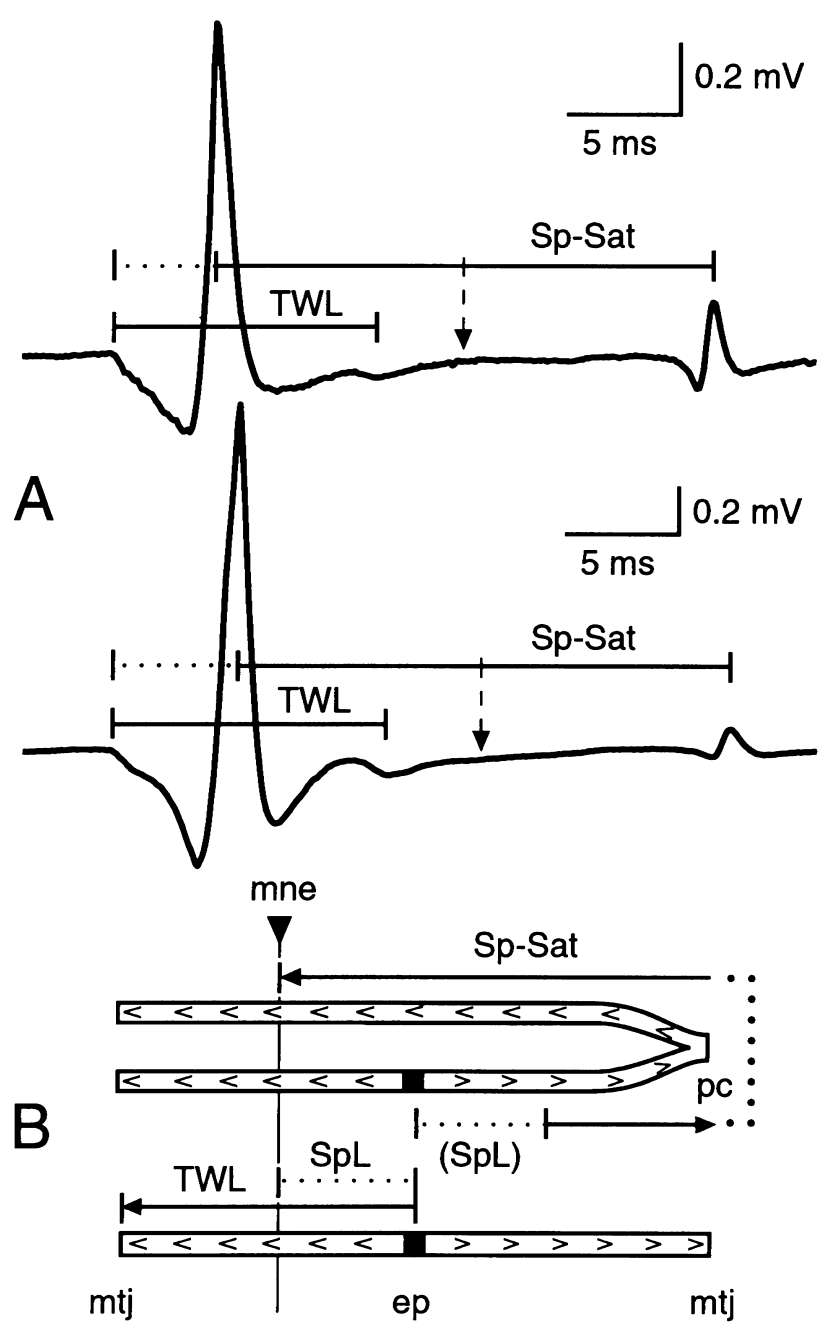

Fig. 4. In 4 of the MUAPs, the spike-satellite interval was approximately twice the terminal-wave latency. (A) Example MUAPs from BB (top) and TA (bottom). (B) Schematic representation of the proposed physiological model, as in Fig. 3. In this case, the electrode is located on the other side of the endplate from the point of continuity, so the satellite latency corresponds to two times the endplate-to-tendon propagation time (TWL) plus the endplate-to-electrode propagation time $(\mathrm{SpL})$. Therefore, Sp-Sat $=$ $2 *$ TWL.

between the spike-satellite interval and the preceding IDI (Fig. 6). After correcting for this dependency, the jitter values ranged from 13 to $71 \mu \mathrm{s}$ in $\mathrm{BB}$, with 7 of 8 values being less than or equal to $45 \mu \mathrm{s}$, and from 29 to $135 \mu \mathrm{s}$ in TA, with 6 of 9 values being less than $80 \mu \mathrm{s}$. For the other 4 MUAPs, the signal-to-noise ratios were too poor to allow accurate measurement of the spike-satellite-interval variation.

\section{Discussion}

\subsection{Previously proposed mechanisms}

Before accepting that satellite potentials in normal human muscles are due to the hypothesized mechanism, it should be asked whether they can be explained in any other way. Several mechanisms have been previously proposed as possible sources of origin for late-latency MUAP components.

One proposed mechanism is that satellite potentials come from newly innervated muscle fibers. Their long latency is explained by the slow conduction velocity in the immature nerve branch, which is initially unmyelinated and of small diameter. However, even if the conduction velocity along the immature nerve branch were as slow as $1.5-2 \mathrm{~m} / \mathrm{s}$ (Uncini et al., 1990), the branch would have to be up to 33-44 mm in length to account for the spike-satellite intervals we observed, which were up to $21.9 \mathrm{~ms}$. The existence of such long axon collaterals in a normal healthy muscle is very unlikely. Moreover, the fact that none of the 21 MUAPs we observed had more than one satellite argues against the possibility that the satellite came from a reinnervated fiber that had been denervated due to loss of a motor axon. If that had been the case, then the reinnervating axon might have been expected to also reinnervate some of the other muscle fibers belonging to the denervated MU, resulting in a MUAP with multiple late components as seen in some neurogenic disorders.

The long latency of satellite potentials has also been attributed to increased neuromuscular transmission delay

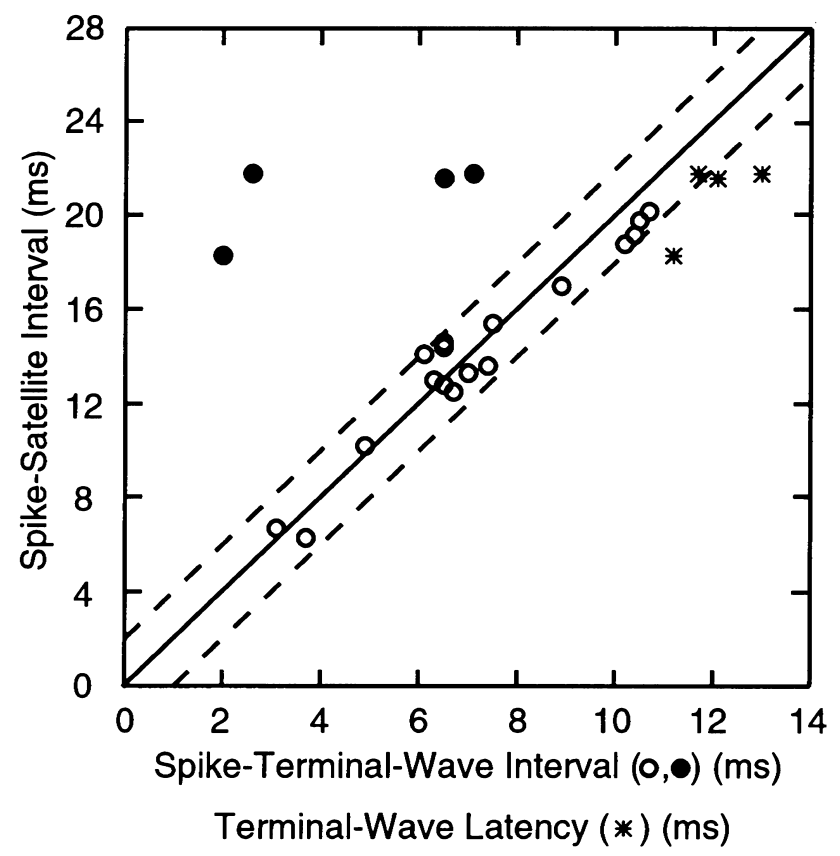

Fig. 5. Spike-satellite intervals of all the MUAPs. For the first group of MUAPs, the spike-satellite interval was approximately twice the spiketerminal-wave interval (open circles), while for the second group it was much larger (filled circles). For all the MUAPs in the first group, the midpoint of the spike-satellite interval fell within $\pm 1 \mathrm{~ms}$ of peak of the terminal wave (dashed lines), which was well within the extent of the terminal wave. For the MUAPs in the second group, the spike-satellite interval was approximately equal to twice the terminal-wave latency (asterisks). 

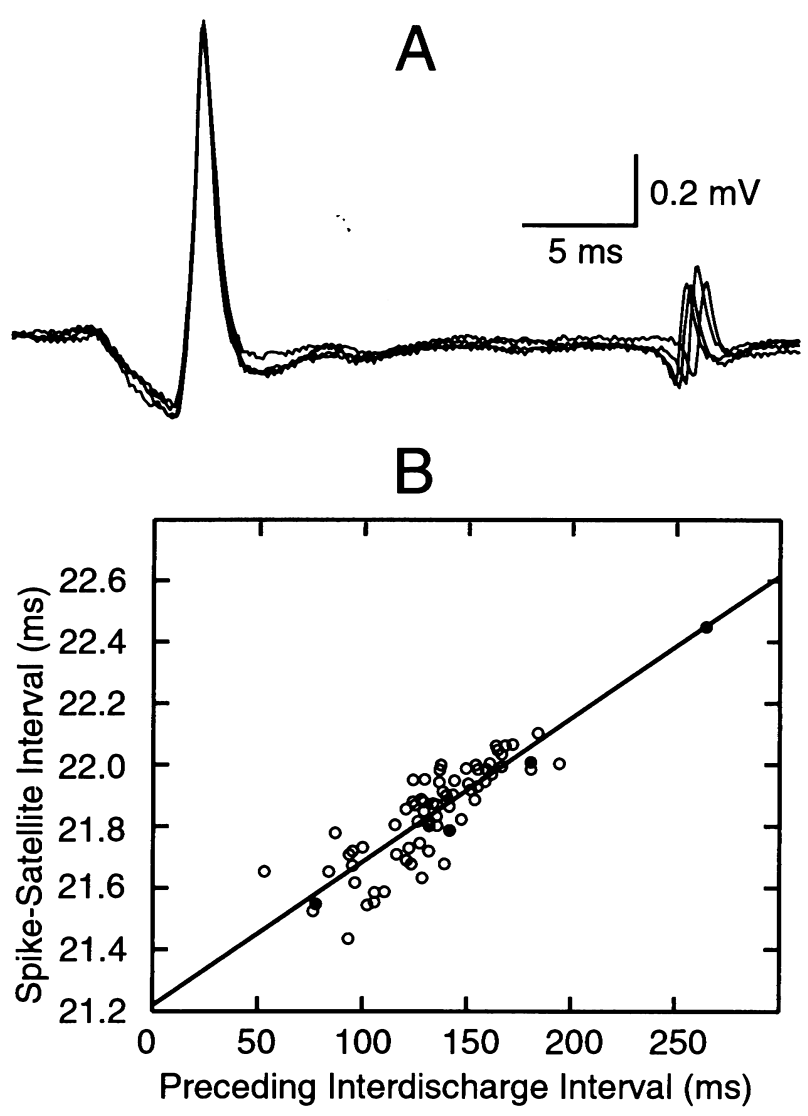

Fig. 6. Positive correlation between the spike-satellite interval and the preceding interdischarge interval (IDI). (A) Four different discharges of a MUAP from BB showing the variability of the spike-satellite interval. (B) Spike-satellite intervals for all 76 discharges of this MUAP versus preceding IDI and the regression line. The 4 discharges shown in (A) are marked as filled circles.

at a newly formed endplate. However, since the normal neuromuscular transmission delay is less than $1 \mathrm{~ms}$, it is very unlikely that this mechanism could account for the long spike-satellite intervals we observed. The lack of blocking and the small spike-satellite-interval variability also argue against an abnormality of neuromuscular transmission.

Late components have also been attributed to an abnormally wide scatter of the endplates along the muscle fibers of the MU. The spatial scatter of the MU endplates is thought to account for the multiple peaks seen in some MUAP spikes (Stålberg and Dioszeghy, 1991; Lateva and McGill, 1998b), each peak being generated by a fraction of the MU innervated by a separate branch of the nerve fiber. In the case of multiple MUAP peaks, the temporal separation between the peaks is never more than about $5 \mathrm{~ms}$, which corresponds to a spatial separation between endplate zones of 15-20 mm (assuming a muscle-fiber conduction velocity of 3-4 m/s). However, to account for a satellite potential such as the one shown in Fig. 1, which has a spike-satellite interval of $19.2 \mathrm{~ms}$, a spatial separation of 58-77 mm would be required. This is twice as long as the MU's endplate-to- tendon distance (based on the MUAP's terminal-wave latency of $10 \mathrm{~ms}$ ), which is unrealistic.

Another proposed mechanism involves slow conduction velocity along atrophic, fragmented, or regenerating muscle fibers. However, Lang and Partanen (1976) measured conduction velocities of satellite potentials in normal muscle and found them to be in the normal range. Moreover, if satellite potentials resulted from abnormally slow muscle-fiber conduction velocity, then one would expect a strong correlation between spike latency and spike-satellite interval. That is, when the recording electrode is farther from the endplate, one would expect a greater difference between the arrival times of the spike and the satellite due to the longer propagation distance. However, our results did not reveal such a correlation (Fig. 2). For example, the MUAP in Fig. 1 had a short spike latency $(1.1 \mathrm{~ms})$, indicating that the recording electrode was very close to the endplate, but a very long satellite latency $(21.4 \mathrm{~ms})$. The satellite conduction velocity would have to have been only about $0.05 \%$ that of the spike, or about $0.15-0.2 \mathrm{~m} / \mathrm{s}$, to produce so long a latency. Such slow muscle-fiber conduction velocities have never been observed.

Finally, Lang and Partanen (1976) suggested that satellite potentials could be related to late components known as axon reflexes which are sometimes seen following nerve stimulation (Fullerton and Gilliatt, 1965; Stålberg and Trontelj, 1970). These components have been attributed to the existence of an axonal branching point located proximal to the site of stimulation. A submaximal stimulus may excite only one of the branches, generating an orthodromic signal that produces the main response, and a signal that travels antidromically to the branching point and then orthodromically along the second branch to produce the late component. However, such a mechanism would not be expected to operate during voluntary activation, since the descending nerve signal would activate both branches.

Thus, even though some or all of those previously proposed mechanisms might provide plausible explanations for late components in pathological muscle, none of them is sufficient by itself to explain the occurrence of the satellite potentials we recorded in normal muscle.

\subsection{Split-fiber mechanism}

We hypothesize that satellite potentials in a normal human muscle are due to an action potential propagating along a non-innervated muscle fiber which is connected to an innervated muscle fiber at one of its muscle/tendon junctions. The long latency of the satellite potential thus reflects the sum of the propagation time along the innervated fiber from the endplate to the muscle/tendon junction, plus the propagation time along the non-innervated fiber from the muscle/tendon junction back to the recording site. This mechanism is consistent with all the results of the present study as well as with the published results from other experimental studies. 


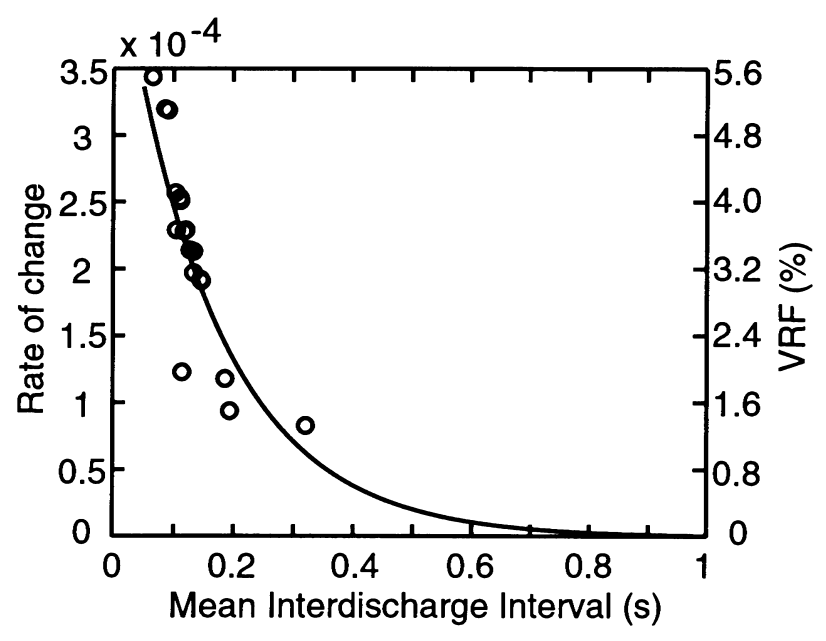

Fig. 7. The slope of the regression line (rate of change) of the spike-satellite interval with respect to the preceding IDI (normalized by the mean value of the spike-satellite interval), plotted as a function of the mean IDI. The MUAP shown in Fig. 6 is marked as a filled circle. The time constant of the exponential fit of the data is $160 \mathrm{~ms}$. The right axis shows recalculated VRF.

Lang and Partanen (1976) used a pair of electrodes with a fixed interelectrode separation to measure the velocity and direction of propagation of both the MUAP main spike and the satellite potential in normal muscle. They found that the main spike and the satellite had similar velocities, but that some satellites propagated in the opposite direction to the spike while others propagated in the same direction (Lang and Partanen, 1976, Fig. 2). This result is consistent with the proposed model when the recording electrode is located on the same (Fig. 3B) or opposite (Fig. 4B) side of the endplate as the point of continuity, respectively.

Pairs of connected muscle fibers could come about by the process of longitudinal muscle-fiber splitting, which has been observed to occur in normal muscle (Bell and Conen, 1968; Swash and Schwartz, 1977; Editorial, 1978; Swash and Schwartz, 1984; Loughlin, 1993). Longitudinal muscle-fiber splitting is generally recognized in transverse sections as a fiber with a deep invagination of its sarcolemma directed toward a centrally located nucleus (Bell and Conen, 1968; Swash and Schwartz, 1977, 1984). Muscle fiber nuclei are normally located just beneath the sarcolemma, but Bell and Conen (1968) found that many normal muscle fibers have centrally located nuclei near their insertions into fascicle sheets and tendons. They concluded that in normal muscle, forking of muscle fibers occurs only in the vicinity of myotendinous junctions.

The sequence of events that take place during longitudinal fiber splitting is not known. Our results suggest that the outcome is a stable configuration in which two daughter fibers have been formed which are completely separate except at one myotendinous junction. One of the daughter fibers lacks an endplate of its own but survives because of its connection to the other daughter fiber, the two in essence constituting one long fiber.
The exact factors that trigger longitudinal fiber splitting in normal muscles are also not known, but muscular hypertrophy is believed to be involved (Swash and Schwartz, 1977; Kakulas and Adams, 1985). Hypertrophy denotes an increase in muscle size because of enlarged myofibrils. Hypertrophy occurs in normal skeletal muscle as an adaptation to physical stress from work or exercise (Ho et al., 1980; Salleo et al., 1980; Antonio and Gonyea, 1993). Theoretical considerations suggest that after fibers enlarge to a certain size, they must undergo splitting in order to maintain a normal surface-area-to-fiber-volume ratio. Splitting of a large parent fiber would mean that the daughter fibers would have nearly normal diameters, which would explain why the main MUAP spike and the satellite have similar conduction velocities (Lang and Partanen, 1976). Whether satellite potentials occur with greater frequency in physically stressed subjects needs further investigation.

It should be mentioned that while the split-fiber mechanism has been considered here in conjunction with healthy muscle, it could be responsible for some of the satellite potentials observed in pathological muscle as well.

\subsection{Spike-satellite-interval variation}

The small but persistent variability of the spike-satellite interval can be attributed to two factors: the velocity recovery function and neuromuscular transmission jitter.

The velocity recovery function (VRF) describes the dependency of muscle-fiber conduction velocity on the preceding discharge history (Stålberg, 1966, 1977; Mihelin et al., 1991). The VRF predicts the conduction velocity to be somewhat higher for shorter preceding IDIs than for longer ones, which explains the positive correlation between spikesatellite interval and preceding IDI (Fig. 6). For the range of IDI values in our study, the VRF further predicts that the spike-satellite interval should have a decreasing exponential relationship to IDI. To confirm this, we plotted the slope of each MUAP's spike-satellite-interval-to-IDI regression line (Fig. 6B), normalized by its mean spike-satellite interval, versus its mean IDI. The results are shown in Fig. 7. They are fit reasonably well by an exponential function with a time constant of $160 \mathrm{~ms}$. Knowing the time constant allows the slope values to be converted to VRF values. The results indicate that the satellite conduction velocities were supernormal by from $5.5 \%$ to $1 \%$ compared to the velocity that would be expected after an IDI of at least $1 \mathrm{~s}$. Both this magnitude and this time constant for the VRF are in substantial agreement with published data (Stålberg 1966, 1977; Mihelin et al., 1991).

The second component of the spike-satellite-interval variability can be attributed to normal neuromuscular transmission jitter. The interval between two SFAPs belonging to the same motor unit exhibits a small variability due to the stochastic nature of the neuromuscular transmission delay (Sanders and Stålberg, 1996). When we computed the mean consecutive difference between the spike-satellite intervals, 
using the method of Davis et al. (1988) to correct for the VRF effects, the values for all but 5 of the satellites fell within the normal range ( $\leq 45 \mu$ s for $\mathrm{BB}, \leq 79 \mu$ s for TA) (Sanders and Stålberg, 1996).

If the MUAP spike were due primarily to the single-fiber action potential of the innervated fiber of the connected pair, then one might expect a very small spike-satellite-interval jitter, since both the MUAP spike and the satellite potential would be activated by the same endplate. Indeed, SFAP pairs with jitters of less than $4 \mu \mathrm{s}$ are occasionally encountered in single-fiber EMG (SFEMG) studies and are considered evidence of the existence of split fibers that are activated by the same endplate via a direct electrical connection (Ekstedt and Stålberg, 1969; Sanders and Stålberg, 1996). We did not observe such low jitter values. There are several possible explanations for this, as well as for the 5 abnormally large jitters we found. First, since our signals were recorded using monopolar needle electrodes, the MUAP spikes probably represented multiple SFAPs rather than a single SFAP. Also, the potentials were broader than they would have been if they had been recorded by a SFEMG electrode, making measurement of the spike-satellite intervals less precise. We estimate that our measurement errors were between $18 \mu \mathrm{s}$ and $125 \mu \mathrm{s}$, making it impossible to detect very small values of jitter. Furthermore, the conduction times in this study were many times longer than those encountered in SFEMG studies, which would amplify any conduction irregularities (Trontelj et al., 1990). It is also possible that our analysis did not completely correct for the VRF effects.

Thus, the results related to spike-satellite-interval variation provide a further confirmation of our hypothesis that the satellite potential is activated by normal neuromuscular transmission and propagates along a normal muscle fiber. They also provide a possible new approach for studying the VRF.

\section{Acknowledgements}

This study was supported by the Medical and Rehabilitation Research and Development Services of the US Department of Veterans Affairs.

\section{References}

Antonio J, Gonyea WJ. Progressive stretch overload of skeletal muscle results in hypertrophy before hyperplasia. J Appl Physiol 1993;75:1263-1271.

Bell CD, Conen PE. Histopathological changes in Duchenne muscular dystrophy. J Neurol Sci 1968;7:529-544.

Borenstein S, Desmedt JE. Range of variations in motor unit potentials during reinnervation after traumatic nerve lesions in humans. Ann Neurol 1980;8:460-467.

Borenstein S, Desmedt JE. Late components of the motor unit potential in muscle diseases. In: Kunze K, Desmedt JE, editors. Studies on neuromuscular diseases, Basel: Karger, 1975. pp. 103-107.

Buchthal F, Guld C, Rosenfalck P. Innervation zone and propagation velocity in human muscle. Acta Physiol Scand 1955;35:174-190.
Buchthal F, Rosenfalck A. On the structure of motor units. In: Desmedt JE, editor. New developments in electromyography and clinical neurophysiology vol. 1, Basel: Karger, 1973. pp. 71-85.

Cruz-Martinez A, Lopez-Terradas JM. Motor unit remodelling in Duchenne muscular dystrophy. Electrophysiological assessment. Electromyogr clin Neurophysiol 1992;32:351-358.

Daube JR. AAEM minimonograph \#11: Needle examination in clinical electromyography. Muscle Nerve 1991;14:685-700.

Davis GR, Ingram DA, Fincham WF, Swash M, Schwartz MS. Jitter correction: a computer algorithm for reduction of the velocity recovery function artifact. Muscle Nerve 1988;11:534-539.

Dimitrova NA, Dimitrov GV, Lateva ZC. Influence of the fiber length on the power spectrum of single muscle fiber extracellular potentiais. Electromyogr Clin Neurophysiol 1991;31:387-398.

Dimitrova N. Model of the extracellular potential field of a single striated muscle fibre. Electromyogr clin Neurophysiol 1974;14:53-66.

Editorial, Muscle fibre splitting: a reappraisal. Lancet 1978;1:646.

Ekstedt J, Stålberg E. Abnormal connections between skeletal muscle fibers. Electroenceph clin Neurophysiol 1969;27:607-609.

Finsterer J, Mamoli B. Satellite potentials as a measure of neuromuscular disorders. Muscle Nerve 1997;20:585-592.

Fullerton PM, Gilliatt RW. Axon reflexes in human motor nerve fibres. J Neurol Neurosurg Psychiatry 1965;28:1-11.

Gootzen TH, Stegeman DF, Van Oosterom A. Finite limb dimensions and finite muscle length in a model for the generation of electromyographic signals. Electroenceph clin Neurophysiol 1991;81:152-162.

Gydikov A, Kosarov D. Extraterritorial potential field of impulses from separate motor units in human muscles. Electromyogr clin Neurophysiol 1972;12:283-305.

Ho KW, Roy RR, Tweedle CD, Heusner WW, Van Huss WD, Carrow RE. Skeletal muscle fiber splitting with weight-lifting exercise in rats. Am J Anat 1980;157:433-440.

Howard JE, McGill KC, Dorfman U. Properties of motor unit action potentials recorded with concentric and monopolar needle electrodes: ADEMG analysis. Muscle Nerve 1988;11:1051-1055.

Kakulas BA, Adams RD. Diseases of muscle. Pathological foundations of clinical myology, fourth ed.. Philadelphia: Harper \& Row, 1985.

Kosarov D, Gydikov A. The influence of the volume conduction on the shape of the action potentials recorded by various types of needle electrodes in normal human muscle. Electromyogr clin Neurophysiol 1975;15:319-335.

Lang AH, Partanen VSJ. 'Satellite potentials' and the duration of motor unit potentials in normal, neuropathic and myopathic muscles. J Neurol Sci 1976;27:513-524.

Lateva ZC, McGill KC. The physiological origin of the slow afterwave in muscle action potentials. Electroenceph clin Neurophysiol 1998a;109: $462-469$.

Lateva ZC, McGill KC. Electromyographic imaging of motor unit architectural properties. Proc 12th ISEK Congr., Montreal, Canada. 1998b: 274-275.

Lateva ZC, Dimitrova NA, Dimitrov GV. Effect of recording electrode position along a muscle fiber on surface potential power spectrum. J Electromyogr Kinesiol 1993;3:195-204.

Lateva ZC, McGill KC, Burgar CG. Anatomical and electrophysiological determinants of the human thenar compound muscle action potential. Muscle Nerve 1996;19:1457-1468.

Loughlin M. Muscle biopsy: a laboratory investigation, Oxford: Butterworth-Heinemann, 1993.

McGill KC, Dorfman LJ. High-resolution alignment of sampled waveforms. IEEE Trans Biomed Eng 1984;BME-31:462-468.

McGill KC, Cummins KL, Dorfman LJ. Automatic decomposition of the clinical electromyogram. IEEE Trans Biomed Eng 1985;BME-32:470 477.

McGill KC, Dorfman LJ. Automatic decomposition electromyography (ADEMG): methodologic and technical considerations. In: Desmedt JE, editor. Computer-aided electromyography and expert systems, Amsterdam: Elsevier, 1989. pp. 91-101. 
Mihelin M, Trontelj JV, Stålberg E. Muscle fiber recovery functions studied with double pulse stimulation. Muscle Nerve 1991;14:739-747.

Roeleveld K, Stegeman DF, Vingerhoets HM, Van Oosterom A. Motor unit potential contribution to surface electromyography. Acta Physiol Scand 1997;160:175-183.

Rowinska-Marcinska K, Ryniewicz B, Hausmanowa-Petrusewicz I, Karwanska A. Diagnostic value of satellite potentials in clinical EMG. Electromyogr clin Neurophysiol 1997;37:483-489.

Salleo A, Anastasi G, La Spada G, Falzea G, Denaro MG. New muscle fiber production during compensatory hypertrophy. Med Sci Sports Exerc 1980;12:268-273.

Sanders DB, Stålberg EV. AAEM minimonograph \#25: Single-fiber electromyography. Muscle Nerve 1996;19:1069-1083.

Stålberg E, Dioszeghy P. Scanning EMG in normal muscle and in neuromuscular disorders. Electroenceph clin Neurophysiol 1991;81:403416.

Stålberg E. Electrogenesis in human dystrophic muscle. In: Rowland LP, editor. Pathogenesis of human muscular dystrophies, Amsterdam: Excerpta Medica, 1977. pp. 570-587.

Stålberg E, Trontelj JV. Demonstration of axon reflexes in human motor nerve fibres. J Neurol Neurosurg Psychiatry 1970;33:571-579.
Stålberg E, Andreassen S, Falck B, Lang H, Rosenfalck A, Trojaborg W. Quantitative analysis of individual motor unit potentials: a proposition for standardized terminology and criteria for measurement. J Clin Neurophysiol 1986;3:313-348.

Stålberg E, Nandedkar SD, Sanders DB, Falck B. Quantitative motor unit potential analysis. J Clin Neurophysiol 1996;13:401-422.

Stålberg E. Propagation velocity in human muscle fibers in situ. Acta Physiol Scand. 1996;70(Suppl 287):1-112.

Swash M, Schwartz MS. Implications of longitudinal muscle fibre splitting in neurogenic and myopathic disorders. J Neurol Neurosurg Psychiatry 1977;40:1152-1159.

Swash M, Schwartz MS. Biopsy pathology of muscle, London: Chapman and Hall, 1984.

Trontelj JV, Stålberg EV, Mihelin M. Jitter in the muscle fiber. J Neurol Neurosurg Psychiatry 1990;53:49-54.

Uncini A, Lange DJ, Lovelace RE, Solomon M, Hays AP. Long-duration polyphasic motor unit potentials in myopathies: a quantitative study with pathological correlation. Muscle Nerve 1990;13:263-267. 